\title{
Spin waves and magnetic anisotropy in ultrathin (111)-oriented cubic films
}

\author{
G. Gubbiotti, G. Carlotti ${ }^{\circ}$ \\ Unità INFM - Dipartimento di Fisica dell'Università, Via Pascoli, $6100 \quad$ Perugia, Italy \\ B. Hillebrands \\ Universität Kaiserslautern, Erwin-Schrödinger-Str. 56, 67663 Kaiserslautern, Germany
}

\begin{abstract}
The dispersions of dipolar (Damon-Eshbach modes) and exchange dominated spin waves are calculated for in-plane magnetized thin and ultrathin cubic films with (111) crystal orientation and the results are compared with those obtained for the other principal planes. The properties of these magnetic excitations are examined from the point of view of Brillouin light scattering experiments. Attention is paid to study the spin-wave frequency variation as a function of the magnetization direction in the film plane for different film thicknesses. Interface anisotropies and the bulk magnetocrystalline anisotropy are considered in the calculation. A quantitative comparison between an analytical expression obtained in the limit of small film thickness and wave vector and the full numerical calculation is given.
\end{abstract}

\section{Introduction}

The study of spin waves in magnetic films with cubic symmetry has shown to be very useful for determining magnetic anisotropy constants. Most of previous studies, however, have only considered (100) and (110) oriented films $^{1-8}$. For the (111) orientation spin-wave frequency calculations have not been presented so far probably due to the more complicated algebra involved and due to the lack of experimental data. Recently an appreciable directional inplane dependence of the spin-wave frequency has been observed by Ferromagnetic Resonance (FMR) in thin Fe (111) films and $\mathrm{Fe}(111) / \mathrm{Cu}(111)$ multilayers ${ }^{9}$ as well as by Brillouin Light Scattering ${ }^{10}$ (BLS) in ultrathin $\mathrm{Ni}$ films. This latter technique, which is based on the inelastic scattering of photons by thermally excited spin waves (thermal magnons), has proved to be a very powerful tool for investigating magnetic properties in magnetic films and multilayers through the detection of spin waves with nonzero wave vector. From BLS measurements of the spinwave frequencies as a function of the direction and magnitude of the in-plane wave vector, $q_{/ /}$, and the direction and strength of the externally applied field, the determination of magnetic parameters such as the in-plane anisotropy constants can be attained. The capability of BLS to determine the interface anisotropy constants is particularly relevant for the (111)-plane of a cubic crystal. This is because torque measurements, like BLS, are always more sensitive to high order anisotropies, like the 6-fold in the (111) orientation, than static methods which yield at the best a very weak directional dependence of the free energy on the (111) plane since, for an in-plane magnetized film, the first order magnetocrystalline anisotropy gives an isotropic contribution ${ }^{11}$.

The aim of this paper is to fill the gap existing in the literature concerning the characteristics of the spin-wave spectrum of thin and ultrathin magnetic films with (111) orientation. The very new experimental results obtained by $\mathrm{BLS}^{10}$, which show an appreciable in-plane directional dependence of the spin-wave frequency on the $\mathrm{Ni}$ (111) plane, and the necessity to interpret them in terms of the anisotropy constants have motivated the theoretical study we are presenting in the following paragraphs. The spin-wave frequency is calculated in the dipole-exchange regime assuming an in-plane magnetized $\mathrm{Ni}$ film and including both interface and bulk anisotropy. This permits us to obtain the frequency dispersion as a function of both the film thickness and the propagation direction on the surface plane.

\section{The calculation procedure}

In order to calculate the spin-wave frequency in the case of an in-plane magnetized (111)-oriented cubic film, we make reference to a continuum model previously developed by one of us for (100) and (110)-oriented films and we are using the same type of nomenclature. ${ }^{1}$ The geometry is defined such that the $\mathrm{x}$ axis is normal to the film interfaces at $\mathrm{x}=0$ (upper interface) and $\mathrm{x}=-\mathrm{L}$ (lower interface), while the applied field is taken along the $\mathrm{z}$ axis. The film is considered to be infinite in the plane and the spin-wave propagation is assumed to be perpendicular to the applied field with a wave vector $\mathrm{q}_{/ /}$defined by the light scattering geometry. The calculation relies upon resolving the equation of motion (linearized Landau-Lifshitz torque equation and the magnetostatic Maxwell equations) in the magnetic layer with appropriate boundary conditions. Inclusion of terms resulting from exchange interaction yields six solutions for the dynamic components of the fields, which are classified according to the wave vector component perpendicular to the layer $\left(q_{x i}, \mathrm{i}=1,2 \ldots 6\right){ }^{1}$ From the magnetic and Rado-Weertman boundary conditions at the film interfaces, a system of 8 linear homogeneous equations in the fluctuating fields inside $\left(h_{x i}\right)$ and outside the layer $\left(h_{x 1}^{e}\right.$ and $h_{x 2}^{e}$ ) is obtained. The problem of finding solutions for the propagating spin waves is therefore reduced to finding the zeros of the $8 \times 8$ boundary condition determinant. A computer program has been written to search for the frequencies that correspond to the roots of this determinant. Although this is a standard 
and well established procedure, it should be noted that the calculations are a little bit more complicated when the (111) crystal orientation is considered, because of the additional contributions in the secular equation given by the magnetocrystalline and interface anisotropy fields $H_{\gamma}$ and $H_{\gamma^{\prime}}$ (see below), which are zero in the (100) and (110) orientations.

In the following we distinguish between volume and interface anisotropy contributions. The former contains the magnetocrystalline anisotropy which originates from the coupling of the magnetization to the crystallographic symmetry, while the latter is due to the lack of translational symmetry along the film normal. These anisotropy fields shift the magnetic excitation frequency. We point out that in this paper we only consider cubic anisotropy, while the possible presence of non-cubic terms, such as uniaxial inplane anisotropy of magnetoelastic origin, is not taken into account.

For cubic crystals and referring to a carthesian coordinate system aligned with the principal crystallographic axes, the free energy associated with the volume anisotropy, $\mathrm{E}_{a n i}$, is defined as

$$
E_{\text {ani }}=K_{1}\left(\alpha_{x}^{2} \alpha_{y}^{2}+\alpha_{x}^{2} \alpha_{z}^{2}+\alpha_{y}^{2} \alpha_{z}^{2}\right)
$$

where the $\alpha_{i}, i=x, y, z$, are the direction cosines of the magnetization relative to the crystallographic axes and $K_{1}$ is the first non-vanishing order magnetocrystalline anisotropy constant in a cubic system. If spherical coordinates are introduced, the explicit forms for $\mathrm{E}_{a n i}$ relative to the three principal crystal orientations are: ${ }^{1,12}$

(100)

$$
E_{\text {ani }}=K_{1}\left(\cos ^{2} \theta \sin ^{2} \theta+\sin ^{4} \theta \cos ^{2} \phi \sin ^{2} \phi\right)
$$

(110)

$$
\begin{aligned}
E_{\text {ani }}= & \frac{K_{1}}{4}\left\{\cos ^{4} \theta+\sin ^{4} \theta\left[\sin ^{4} \phi+\sin ^{2}(2 \phi)\right]\right. \\
& \left.+\sin ^{2}(2 \theta)\left[\cos ^{2} \phi-\frac{1}{2} \sin ^{2} \phi\right]\right\}
\end{aligned}
$$

(111)

$$
\begin{aligned}
E_{a n i} & =K_{1}\left\{\frac{1}{3} \cos ^{4} q+\frac{1}{4} \sin ^{4} q\right. \\
& \left.-\frac{\sqrt{2}}{3} \sin ^{3} \theta \cos \theta \cos (3 \phi)\right\}
\end{aligned}
$$

The azimuthal angle $\phi$, which defines the magnetization direction in the film plane, is measured with respect to the crystallographic (001) axis of the layer plane for the (100) and the (110) orientations while for the (111) orientation it is measured with respect to the $(\overline{1} 10)$ axis. $\theta$ is the polar angle.

Concerning the interface free energy, $E_{\text {inter }}$, it can be expressed in lowest symmetry consistent with the symmetry of the respective surface plane as:

(100)

$$
E_{\text {inter }}=-k_{s} \cos ^{2} \theta+k_{p} \sin ^{4} \theta \cos ^{2} \phi \sin ^{2} \phi
$$

$$
E_{\text {inter }}=-k_{s} \cos ^{2} \theta+k_{p} \sin ^{2} \theta \cos ^{2} \phi
$$

(111)

$$
E_{\text {inter }}=-k_{s} \cos ^{2} \theta-k_{p} \frac{\sqrt{2}}{3} \sin ^{3} \theta \cos \theta \cos (3 \phi)
$$

In Eqs. 5-7, $k_{\mathrm{s}}$ is the out-of-plane anisotropy constant while $k_{\mathrm{p}}$ is the first non-vanishing term of the in-plane interface anisotropy. For $k_{\mathrm{S}}>0$ the surface normal is an easy axis, for $k_{\mathrm{S}}<0$ the film plane is an easy plane of interface anisotropy. Please note that eqs. 5-7 are similarly defined as the projection of the bulk anisotropies on the respective surface plane. The above expressions of the in-plane interface free energy are not unique, in the sense that one can also use other formulations, provided that the crystal in-plane symmetry is correctly taken into account. We would like to emphasize that the expressions for the interface free energy (Eqs. 5-7) need to describe the dependence on the polar angle $\theta$ correctly, since due to the precession of the moments an out-of-plane component in the dynamic magnetization exists which interacts with the out-of-plane anisotropy field components $\mathrm{H}_{\alpha}$ and $\mathrm{H}_{\beta}$ (see below).

The volume $\left(\mathrm{H}_{\alpha}, \mathrm{H}_{\beta}\right.$ and $\left.\mathrm{H}_{\gamma}\right)$ and interface $\left(\mathrm{H}_{\alpha}, \mathrm{H}_{\beta}\right.$, and $\mathrm{H}_{\gamma^{\prime}}$ ) anisotropy fields for an in-plane magnetized film $\left(\theta=90^{\circ}\right)$ can be easily obtained from Eqs. 2-7 applying the following formulas ${ }^{8}$ :

$$
\begin{array}{rlrl}
H_{\alpha} & =\frac{\partial^{2}}{M \partial \theta^{2}} E_{\text {ani }} & H_{\alpha^{\prime}}=\frac{\partial^{2}}{M \partial \theta^{2}} E_{\text {inter }} \\
H_{\beta}=\frac{\partial^{2}}{M \partial \phi^{2}} E_{\text {ani }} & H_{\beta^{\prime}}=\frac{\partial^{2}}{M \partial \phi^{2}} E_{\text {inter }} \\
H_{\gamma}=\frac{\partial^{2}}{M \partial \theta \partial \phi} E_{\text {ani }} & H_{\gamma^{\prime}}=\frac{\partial^{2}}{M \partial \theta \partial \phi} E_{\text {inter }}
\end{array}
$$

\begin{tabular}{llll}
\hline \hline & $\mathrm{H}_{\alpha}$ & $\mathrm{H}_{\beta}$ & $\mathrm{H}_{\gamma}$ \\
\hline$(100)$ & $-\frac{2}{M}\left(k_{s}+2 k_{p} \cos ^{2} \phi \sin ^{2} \phi\right)$ & $\frac{2 k_{p}}{M}\left(1-8 \cos ^{2} \phi \sin ^{2} \phi\right)$ & 0 \\
$(110)$ & $-\frac{2}{M}\left(k_{s}+k_{p} \cos ^{2} \phi\right)$ & $\frac{2 k_{p}}{M}\left(2 \sin ^{2} \phi-1\right)$ & 0 \\
$(111)$ & $-\frac{2}{M} k_{s}$ & 0 & $-\frac{k_{p}}{M} \sqrt{2} \sin (3 \phi)$ \\
\hline \hline
\end{tabular}

Table I. First non-vanishing interface anisotropy fields for in-plane magnetized (100)-, (110)- and (111)-oriented crystal. 
where $\mathrm{M}$ is the saturation magnetization. The first nonvanishing interface anisotropy fields for the principal planes of a cubic crystal are reported in Table I.

The following theoretical results are a refinement of those reported in Refs. 1 and 8. We do not want to describe each step of the calculation and therefore the reader is invited to inspect the equations listed in the Appendix. When the anisotropy fields $H_{\gamma}$ and $H_{\gamma^{\prime}}$ are taken into account, the secular equation becomes a sixth order polynomial equation in the wave vector component perpendicular to the layers $q_{x}$ rather than a bicubic equation in $q_{x}^{2}$, as used in Ref. 1. Concerning the linear system of eight homogeneous equations, four of them are given by the Rado-Weertman boundary conditions applied at the film interfaces which have to be modified if the (111) crystal orientation is considered, while the remaining four homogeneous equations of the linear system, obtained from the continuity of the parallel component of the field $\vec{h}$ and the normal component of $\vec{h}+4 \pi \vec{m}$ at the film interfaces, remain unchanged. We notice that if the values of $k_{\mathrm{s}}$ and $k_{\mathrm{p}}$ are not equal on the two film interfaces, the anisotropy fields entering in Rado-Weertman boundary conditions are different for the two film interfaces $(x=0$ and $x=-L)$. As shown in Ref. 1, different values of the interface anisotropies on either side of the film result in different spin wave frequencies for the Damon-Eshbach mode and the exchange modes for $q_{/ /}$and $-q_{/ /}$. Therefore in a BLS experiment the spin wave frequencies obtained from the Stokes and the anti-Stokes part of the spectrum may differ in their absolute values. This can be utilized for the separate determination of the interface anisotropy constants of each interface.

\section{Comparison between numerical and analytical calculations}

In the previous section, we have presented the theoretical model we use for the numerical calculation of the spin-waves frequency which are typically observed in a Brillouin light scattering experiment. We note that, when dealing with ultrathin magnetic films where only the Damon-Eshbach spin-wave mode can be detected, the complexity of the numerical calculation can be overcome by use of a more straighforward analytical procedure. Following the approach proposed by Stamps and Hillebrands ${ }^{13}$, one can treat the magnetization as uniform across the magnetic film and if the product of the film thickness $\mathrm{L}$ and the wave vector $q_{/ /}$is small compared to unity, the frequency of the Damon-Eshbach mode for an inplane magnetized sample can be expressed as ${ }^{10}$ :

$$
\begin{array}{r}
\left(\frac{\omega}{\gamma}\right)^{2}=\left[\left(\mathrm{H}_{\mathrm{o}}+\mathrm{H}_{\alpha}+\frac{2}{\mathrm{~L}} \mathrm{H}_{\alpha^{\prime}}+\frac{2 \mathrm{~A}}{\mathrm{M}_{\mathrm{s}}} q_{/ /}^{2}+4 \pi \mathrm{M} f\left(1-\mathrm{q}_{/ /} \mathrm{L} / 2\right)\right) \times\right. \\
\left(\mathrm{H}_{\mathrm{o}}+\mathrm{H}_{\beta}+\frac{2}{\mathrm{~L}} \mathrm{H}_{\beta^{\prime}}+\frac{2 \mathrm{~A}}{\mathrm{M}_{\mathrm{s}}} q_{/ /}^{2}+2 \pi \mathrm{M} f q_{/ /} \mathrm{L} \sin ^{2} \alpha\right)
\end{array}
$$

$$
\left.-\left(\mathrm{H}_{\gamma}+\frac{2}{\mathrm{~L}} \mathrm{H}_{\gamma^{\prime}}\right)^{2}\right]
$$

The parameter $\mathrm{f}$ is the demagnetization factor of ultrathin films, which for $n>1$ is approximatively $\mathrm{f}=1-0.2338 / n$ with $n$ the number of monolayers ${ }^{14}$. Since the magnetization is uniform across the film, the interface torques from the interface anisotropies are converted into volume torques acting on the total film magnetization and the interface anisotropy fields are converted into effective volume anisotropy fields weighed by $2 / \mathrm{L}$, with the factor of two counting the two interfaces of the film. Rado ${ }^{15}$ and Gradmann et al. ${ }^{16}$ give estimates for the range of validity of this assumption. Eq. 11 contains both the volume and the interface anisotropy fields and it can be easily applied to Brillouin scattering experiments. We note that the term $\left(\mathrm{H}_{\gamma}+\frac{2}{L} \mathrm{H}_{\gamma^{\prime}}\right)$ gives a non-vanishing contribution only for a (111)-oriented crystal.

We would now like to discuss the limit of applications of Eq. 11 by means of a comparison to data obtained by using the full numerical procedure. To this respect, Fig. 1 shows the results obtained with the two approaches for the calculation of the spin-wave frequency of (111) oriented Ni films with thicknesses of $60 \AA$ (upper two lines) and $30 \AA$ (lower two lines), respectively. Unless otherwise indicated we use the following magnetic parameters taken from a fit to the experimental data of a (111)-oriented $60 \AA \mathrm{Ni}$ thick film ${ }^{10}$ : $\gamma=1.917 \times 10^{7} \mathrm{~Hz} / \mathrm{Oe}, \quad A=0.73 \times 10^{-6} \mathrm{erg} / \mathrm{cm}$, $4 \pi M=6.03 \mathrm{kOe}$. The value of the applied field is always of $\mathrm{H}=1 \mathrm{kOe}$, the magnitude of the in-plane wave vector is $q_{\|}=1.73 \times 10^{5} \mathrm{~cm}^{-1}$ and the values of the anisotropy

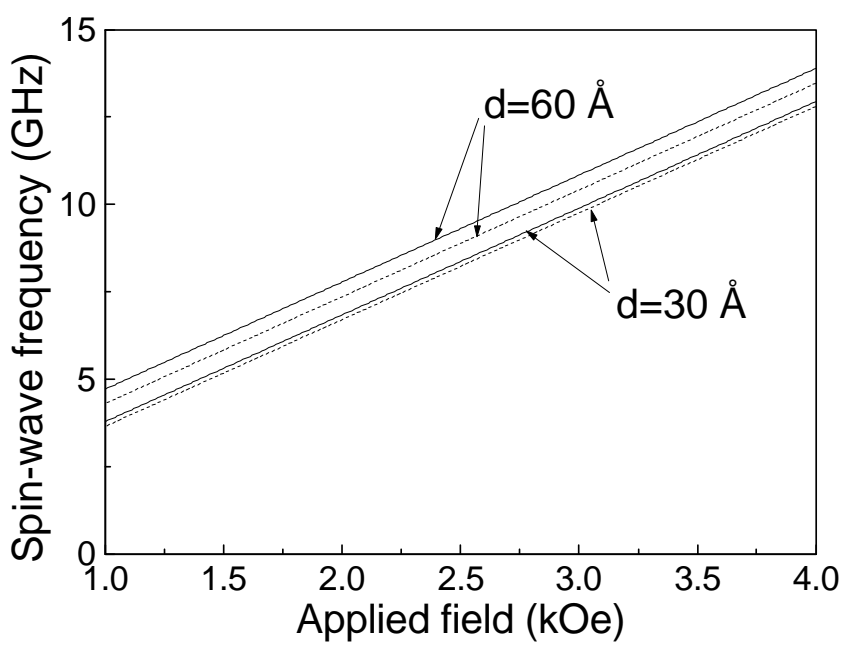

FIG. 1 Magnetic field dependence of the Damon-Eshbach mode frequency for two (111)-oriented, $60 \AA$ and $30 \AA$ thick Ni films. The continuous lines are calculated using Eq. 11 while the dashed lines are calculated by means of the numerical procedure described in the text. For the thicker $\mathrm{Ni}$ film $k_{\mathrm{p}}=0.15 \mathrm{erg} / \mathrm{cm}^{2}$ and $k_{\mathrm{s}}=0.375 \mathrm{erg} / \mathrm{cm}^{2}$, while for the thinner $k_{\mathrm{p}}=0.07 \mathrm{erg} / \mathrm{cm}^{2}$ and $k_{\mathrm{s}}=0.21 \mathrm{erg} / \mathrm{cm}^{2}$. For both Ni film $\mathrm{K} 1=-5.1 \times 10^{4} \mathrm{erg} / \mathrm{cm}^{3}$. 
constants is reported in the caption of Fig.1. For both films the condition $q_{/ /} L<<1$ is verified. The agreement between the analytical (continuous line) and the numerical (dashed line) calculations is, however, better for the thinner Ni film, $q_{/ /} L=0.052$, where the data are almost superimposed, than for the thicker film, $q_{/ /} L=0.104$, where an appreciable frequency difference, which exceeds the Brillouin experimental resolution, is observed. The observed discrepancy of the curves is mainly caused by the approximations made when the terms containing the product $\mathrm{q}_{/ / \mathrm{L}} \mathrm{L}$ are expanded in power series to the first order in the argument $\mathrm{q}_{/ / \mathrm{L}}{ }^{10}$ Using the approximative equation 11 for fitting the experimental data using the anisotropy constant $k_{\mathrm{s}}$ as fit parameter, one would yield a value of $k_{\mathrm{s}}$ which is $7 \%$ greater than the value obtained by fitting the data using the full numerical procedure. Caution must therefore be exercised when using the analytical approach, even for very low film thicknesses.

\section{Results and discussion}

In Fig. 2 we plot the calculated spin-wave frequencies for $\mathrm{Ni}$ (100)-, (110)- and (111)- oriented films as a function of the film thickness. In addition to the surface Damon-Eshbach (DE) mode, a large number of bulk standing modes, characterized by their typical $1 / \mathrm{L}^{2}$ behaviour, are visible. While the bulk modes have almost the same thickness dependence, thus being insensitive to the crystal orientation, differences in the DE spin-wave frequency can be observed when the film thickness is reduced below $200 \AA$. In this condition, interface anisotropy greatly influences the spin-wave frequency giving rise to an appreciable difference between the principal crystal orientations. These frequency differences are due to the fact

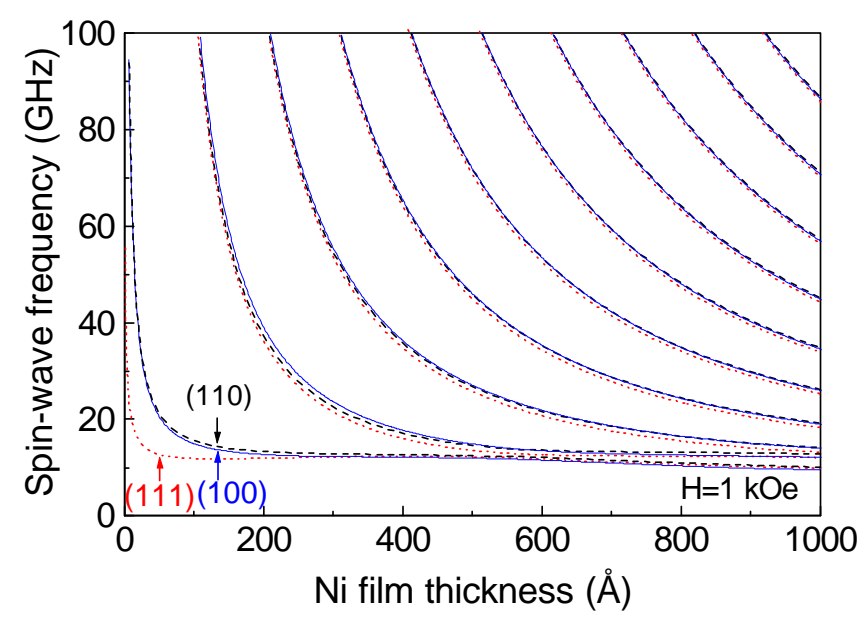

FIG.2 Spin-wave frequency as a function of the layer thickness for a singlecrystal Ni (100)-, (continuous line), (110)-, (dashed line), and (111)-, (dotted line), orientated layer. All the curves are calculated with the full numerical procedure described in the text, taking $\phi=0$ and assuming the out-of-plane anisotropy constants at the two film interfaces to be equal: $k_{\mathrm{s}}=0.375 \mathrm{erg} / \mathrm{cm}^{2}$. that, although the anisotropy constants used to calculate the curves of Fig. 2 are the same for the three crystal orientations, they enter in different ways in the expression for the interface anisotropy fields (see Table I). In particular, it is well known that the large increase of the frequency of the DE mode, which takes place for Ni film thicknesses lower that $60 \AA$, is typical of this interfaceanisotropy-dominated mode ${ }^{3}$. Another interesting aspect of the spin waves dispersion curves is the mode repulsion at the branch crossing where the surface and the bulk spin waves interact interchanging their mode character.

We now analyse in more detail the spin-wave frequency dispersion as the magnetization direction is varied in the film plane. The main differences between cubic films with different crystallographic orientations will be discussed and information will be gained about the best conditions for determining interface anisotropy constants. For the simulations which follow, the applied field is of $1 \mathrm{kOe}$ and we have always kept fixed the volume anisotropy constant to the value ${ }^{17} K_{1}=-5.1 \times 10^{4} \mathrm{erg} / \mathrm{cm}^{3}$ and the outof-plane anisotropy constant to $k_{s}=0.375 \mathrm{erg} / \mathrm{cm}^{2}$. Fig. 3 shows the spin-wave dispersion of the Damon-Eshbach mode as a function of the angle $\phi$ for the principal planes of a $200 \AA$ thick Ni film. All the curves are calculated by means of the full numerical procedure described in Sect. 2, with the in-plane interface anisotropy constant $k_{\mathrm{p}}$ set to zero. Therefore the directional dependence of the spin-wave frequency is only caused by the magnetocrystalline bulk anisotropy in the simulations. Maxima in the spin-wave frequencies indicate easy directions of the magnetization. For the (111)-oriented film, the frequency shows a six-fold periodicity which reflects the in-plane layer symmetry and the amplitude of the frequency oscillation is greatly reduced with respect to those relating to the (100)- and the (110)oriented films. For these last two orientations, the four-fold and two-fould angular periodicity result from the symmetry of the bulk anisotropy fields, respectively. ${ }^{8}$ Our calculations

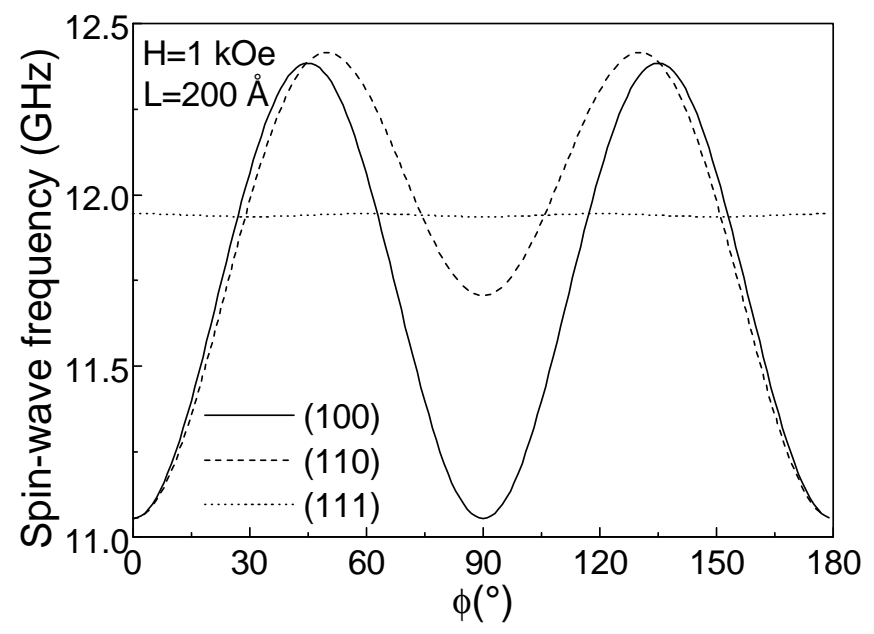

FIG. 3 Spin-wave frequencies for the principal planes of a cubic crystal as a function of the angle $\phi$ for an applied magnetic field of $1.0 \mathrm{kOe}$. The in-plane anisotropy is set to zero on both film interfaces. 
indicate that the experimental determination of the in-plane anisotropy constants from the spin-wave frequency dispersion is very difficult for thick (111)-Ni films since the frequency dispersion with the in-plane angle is comparable with or lower than the typical Brillouin scattering resolution $(\cong 0.3 \mathrm{GHz})$. This type of measurements becomes more feasible in the case of ultrathin films since the interface anisotropy constant $k_{\mathrm{p}}$, converted into an effective volume anisotropy field, yields a large contribution to the angular dispersion of the spin-wave frequency. To illustrate this aspect the calculated angular dispersion of the spin-wave frequency for a (111)-oriented $200 \AA$ thick $\mathrm{Ni}$ film is compared in Fig. 4 with that of $\mathrm{Ni}$ films of lower thicknesses (100 $\AA$ and $50 \AA$ ). The curves are calculated assuming the interface anisotropy constants $k_{p}=0.15 \mathrm{erg} / \mathrm{cm}^{2}$ and $k_{s}=0.375 \mathrm{erg} / \mathrm{cm}^{2}$.

The peak-to-peak frequency amplitude, which essentially depends on $k_{\mathrm{p}}$, increases from about $0.2 \mathrm{GHz}$, for the thick films, to approximatively $1.4 \mathrm{GHz}$, for the a $50 \AA$ thick Ni film. This is within the range of sensitivity of a typical BLS experiment. Results of the analysis of the directional variation of spin-wave frequency of $25 \AA$, $30 \AA$ and $60 \AA$ thick Ni films are reported elsewhere ${ }^{10}$.

\section{Conclusions}

We have studied the dispersion of dipolar (DamonEshbach modes) and exchange dominated spin waves for inplane magnetized thin and ultrathin cubic films with (111) crystal orientation. A quantitative comparison between the results of the complete numerical approach and those of a simplified analytical expression valid in the ultrathin-film limit has shown that even for a Ni film as thin as $60 \AA$ a suitable correction of the out-of-plane anisotropy constant $k_{\mathrm{s}}$ has to be made in order to achieve an acceptable consistency of the data. The results obtained for the (111) orientation have been compared with those relative to the other principal planes of a cubic crystal. It has been shown that

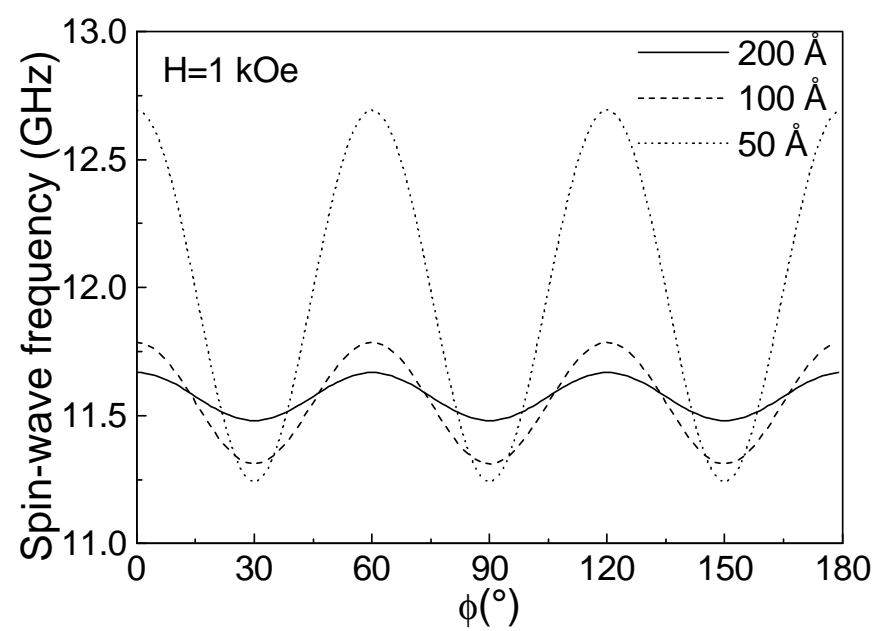

FIG. 4 Spin-wave frequencies for (111)-oriented cubic crystal as a function of the angle $\phi$ for film thickness of $200 \AA, 100 \AA$ and $50 \AA$. while the frequency variation versus the angle $\phi$ in thick films is rather small, this variation increases in ultrathin films because of the greater contribution given by the inplane anisotropy energy which is converted into an effective volume anisotropy. We believe that the results presented in this paper will stimulate further BLS investigations of magnetic anisotropy on the (111)-plane of ultrathin films and multilayers.

\section{Acknowledgments}

G.G. would like to thank the University of Kaiserslautern for the hospitality during a 3 months visit. $\mathrm{He}$ also acknowledges financial support (PhD grant) from the University of Camerino, Italy.

\section{Appendix}

Referring to the equations contained in Ref. 1 as Eq. (1.n) where $\mathrm{n}$ indicates the equation number, we list the refinements of those expressions which enters in the spin wave frequency calculation for a cubic crystal with (111) orientations:

$$
M h_{y}+\left(\frac{i \omega}{\gamma}-H_{\gamma}\right) m_{x}-\left[H+H_{\beta}+2 \frac{A}{M} q^{2}\right] m_{y}=0,
$$

$-M h_{x}+\left[H+H_{\alpha}+2 \frac{A}{M} q^{2}\right] m_{x}+\left(\frac{i \omega}{\gamma}+H_{\gamma}\right) m_{y}=0$,

(1.16)

$\frac{d E_{a n i}}{d \alpha_{x}}=H_{\alpha} m_{x}+H_{\gamma} m_{y}$,

(1.17)

$\frac{d E_{a n i}}{d \alpha_{y}}=H_{\gamma} m_{x}+H_{\beta} m_{y}$,

The secular equation assumes the following form:

(1.22)

$\left[\frac{\omega^{2}}{\gamma^{2}}+H_{\gamma}^{2}-H_{a} H_{b}\right] q^{2}-4 \pi M\left[H_{b} q_{x}^{2}+H_{a} q_{y}^{2}-2 H_{\gamma} q_{x} q_{y}\right]=0$.

It can be converted into a polynomial equation:

$\sum_{i=0}^{6} a_{i} q_{x}^{i}=0$

with coefficients:

$$
\begin{aligned}
& a_{0}=q_{/ /}^{6}+\frac{M}{2 A}\left(2 H+H_{\alpha}+H_{\beta}+4 \pi M\right) q_{/ /}^{4}+\left(\frac{M}{2 A}\right)^{2} \times \\
& \times\left[\left(H+H_{\beta}\right)\left(H+H_{\alpha}+4 \pi M\right)-H_{\gamma}^{2}-8 \pi A q_{z}^{2}-\left(\frac{\omega^{2}}{\gamma^{2}}\right)\right] q_{/ /}^{2}+ \\
& 4 \pi M\left(\frac{M}{2 A}\right)^{2}\left[\left(H_{\alpha}-H_{\beta}\right) q_{/ /}^{2}-\left(H+H_{\alpha}\right) q_{z}^{2}\right],
\end{aligned}
$$


$a_{1}=-8 \pi M H_{\gamma} q_{y}\left(\frac{M}{2 A}\right)^{2}$

$a_{2}=\left\{3 q_{/ /}^{4}+2 q_{/ /}^{2}\left(\frac{M}{2 A}\right)\left(2 H+H_{\alpha}+H_{\beta}+4 \pi M\right)+\left(\frac{M}{2 A}\right)^{2} \times\right.$

$\left.\left[\left(H+H_{\beta}\right)\left(H+H_{\alpha}+4 \pi M\right)-H_{\gamma}^{2}-8 \pi A q_{z}^{2}-\frac{\omega^{2}}{\gamma^{2}}\right]\right\}$

$a_{3}=0$,

$a_{4}=\left[3 q_{/ /}^{2}+\left(\frac{M}{2 A}\right)\left(2 H+H_{\alpha}+H_{\beta}+4 \pi M\right)\right]$,

$a_{5}=0$ and $a_{6}=1$,

$$
\begin{aligned}
& u_{i}=\frac{M}{D}\left[H_{b}-\frac{q_{y}}{q_{x i}}\left(\frac{i \omega}{\gamma}+H_{\gamma}\right)\right], \\
& v_{i}=\frac{M}{D}\left[H_{a} \frac{q_{y}}{q_{x i}}+\left(\frac{i \omega}{\gamma}-H_{\gamma}\right)\right],
\end{aligned}
$$

where $D=H_{a} H_{b}-H_{\gamma}^{2}-\left(\frac{\omega^{2}}{\gamma^{2}}\right)$ and A is the exchange stiffness constant.

The Rado-Weertman boundary conditions calculated at the two film surfaces become:

(1.37) $\sum_{i=1}^{6}\left[\left(M H_{\alpha^{\prime}}-2 A i q_{x i}\right) u_{i}+M H_{\gamma^{\prime}} v_{i}\right] h_{x i}=0$,

(1.37) $\sum_{i=1}^{6}\left[\left(M H_{\alpha^{\prime}}+2 A i q_{x i}\right) u_{i}+M H_{\gamma^{\prime}} u_{i}\right] h_{x i} e^{i q_{x i} L}=0$

(1.38) $\sum_{i=1}^{6}\left[M H_{\gamma^{\prime}} u_{i}+\left(M H_{\beta^{\prime}}-2 A i q_{x i}\right) v_{i}\right] h_{x i}=0$,

(1.38) $\sum_{i=1}^{6}\left[M H_{\gamma^{\prime}} u_{i}+\left(M H_{\beta^{\prime}}+2 A i q_{x i}\right) v_{i}\right] h_{x i} e^{i q_{x i} L}=0$

All the quantities which appear in the previous equations reduce to those reported in Ref. 1 when $H_{\gamma}=0$ and $H_{\gamma^{\prime}}=0$
In the original paper there was a misprint in eq. 34 whose correct form is:

(1.34) $\sum_{i=1}^{6}\left(1+4 \pi u_{i}\right) h_{x i} e^{-i q_{x i} d_{n}}-h_{x 1}^{e} e^{-q_{/ /} d_{n}}-h_{x 2}^{e} e^{q_{/ /} d_{n}}=0$.

\section{REFERENCES}

[1] B. Hillebrands, Phys. Rev. B 41, 530 (1990).

[2] G. Rupp, W. Wettling, R. S. Smith, and W. Jantz, J. Magn. Magn. Mater. 45, 404 (1984).

[3] B. Hillebrands, P. Baugmart, and G. Guntherodt, Phys. Rev B 36, 2450 (1987).

[4] P. Krams, F. Lauks, R. L. Stamps, B. Hillebrands, and G. Guntherodt, Phys. Rev. Lett. 69, 3674 (1992).

[5] R.J. Hichen, D. E. P. Eley, M. Gester, S. J. Gray, C. Daboo, A. J. R. Ives, and J. A. C. Bland, J. Magn. Magn. Mater. 145, 278 (1995).

[6] S. Subramanian, X. Liu, R. L. Stamps, R. Sooryakumar, and G. A. Prinz, Phys. Rev. B 52, 10194 (1995).

[7] X. Liu, M. Steiner, R. Sooryakumar, G. A. Prinz, R. F. C. Farrow, and G. Harp, Phys. Rev. B 53, 12166 (1996).

[8] B. Hillebrands, in Light scattering in Solids VII, edited by M. Cardona and G. Guntherodt, Springer Series in Topics Applied Physics (Springer Verlag, Berlin, in press).

[9] S. M. Rezende, J. A. S. Moura, F. M. de Aguiar, and W. H. Schreiner, Phys. Rev. B 49, 15105 (1994).

[10] G. Gubbiotti, G. Carlotti, G. Socino, F. D’Orazio, F. Lucari, R. Bernardini, and M. De Crescenzi, Phys. Rev. B 56, 11073 (1997)

[11] R. M. Bozorth, Ferromagnetism (IEEE Press, New York, 1993), p. 579.

[12] This expression is directly obtained from the definition of the magnetocrystalline energy of a cubic crystal, Eq. 1, on introducing the direction cosines of the magnetization:

$\alpha_{x}=1 / \sqrt{3}[\cos \theta+\sqrt{2} \sin \theta \cos \phi]$,

$\alpha_{y}=1 / \sqrt{3}[\cos \theta+\sqrt{2} \sin \theta \cos (\phi-2 / 3 \pi)]$,

$\alpha_{z}=1 / \sqrt{3}[\cos \theta+\sqrt{2} \sin \theta \cos (\phi-4 / 3 \pi)]$

in a rotated coordinate system with the $\mathrm{z}^{\prime}$ axis along the (111) direction.

[13] R. L. Stamps, and B. Hillebrands, Phys. Rev. B 44, 12417 (1991).

[14] B. Heinrich, Z. Celinski, J. F. Cochran, A. S. Arrott, and K. Myrtle, Phys. Rev. B 44, 530 (1990).

[15] G. T. Rado, J. Appl. Phys. 61, 4262 (1987).

[16] U. Gradmann, J. Korecki, and G. Waller, Appl. Phys. A 39, 101 (1986).

[17] J. Crangle, in The magnetic properties of matter, (E. Arnold, London, 1977), p.112 